\section{Frequency dependent effect of selective biphasic left vagus nerve stimulation on heart rate and arterial pressure}

\author{
TOMISLAV MIRKOVIC • IVAN KNEŽEVIČ • IVAN RADAN \\ JANEZ ROZMAN • BORUT GERŠAK • MATEJ PODBREGAR
}

MATEJ PODBREGAR $(\bowtie) \bullet$ IVAN RADAN

Centre for Intensive Care Medicine University Clinical Centre Ljubljana Zaloška 4, 1000 Ljubljana SI- Slovenia

Phone: ++38615223182

Fax: ++38615222236 E-mail: matej.podbregar@guest.arnes.si

TOMISLAV MIRKOVIC Clinical Department of Anesthesiology and Intensive Care University Clinical Centre Ljubljana Ljubljana, Slovenia

IVAN KNEŽEVIČ • BORUT GERŠAK

Clinical Department of Cardiovascular Surgery University Clinical Centre Ljubljana Ljubljana, Slovenia

JANEZ ROZMAN Center for Implantable Technology and Sensors ITIS d. o. o. Ljubljana Ljubljana, Slovenia

Trial registration number: NCT 00983632 www.clinicaltrial.gov

\begin{abstract}
Activation of the parasympathetic pathway leads to negative chronotropic, dromotropic, and inotropic changes of heart function. The ability to selectively stimulate certain superficial compartments of peripheral nerves has been demonstrated previously. The aim of the present study was to find a clinically acceptable selective biphasic vagus nerve stimulation technique, which could allow gradual regulation of heart rate and systemic arterial pressure. In two patients, the left vagus nerve was stimulated with a combination of quasi-trapezoidal cathodic and rectangular anodic current pulses with different stimulation frequencies $(10 \mathrm{~Hz}, 20 \mathrm{~Hz}, 30 \mathrm{~Hz})$ and increasing current. The heart rate and systemic arterial pressure decreased with increasing current at all different stimulation frequencies $(p<0.05)$. The heart rate and arterial pressure response was more gradual with $10 \mathrm{~Hz}$ compared to $20 \mathrm{~Hz} / 30 \mathrm{~Hz}$ vagus nerve stimulation $(p<0.05)$. In conclusion, selective vagus nerve stimulation, with a combination of quasi-trapezoidal cathodic and rectangular anodic current pulses at $10 \mathrm{~Hz}$, offers gradual heart rate and systolic arterial pressure control.
\end{abstract}

Key words: vagus nerve, electrical stimulation, heart rate, arterial pressure

\section{Introduction}

The use of nerve stimulation for treating and controlling a variety of medical, psychiatric, and neurological disorders has seen significant growth over the last several decades. In particular, vagal nerve stimulation (VNS) has been the subject of considerable research. (1-3) Currently VNS has numerous potential clinical applications from heart failure to endocrine regulation. The vagus nerve extensively innervates the tho- racic and abdominal viscera (afferent $80 \%$ of fibers) and is an important route of information into the central nervous system (efferent $20 \%$ of fibers). (4) Activation of the parasympathetic pathway leads to negative chronotropic, dromotropic, and inotropic changes of the heart function. $(5,6)$ The vagus nerve 

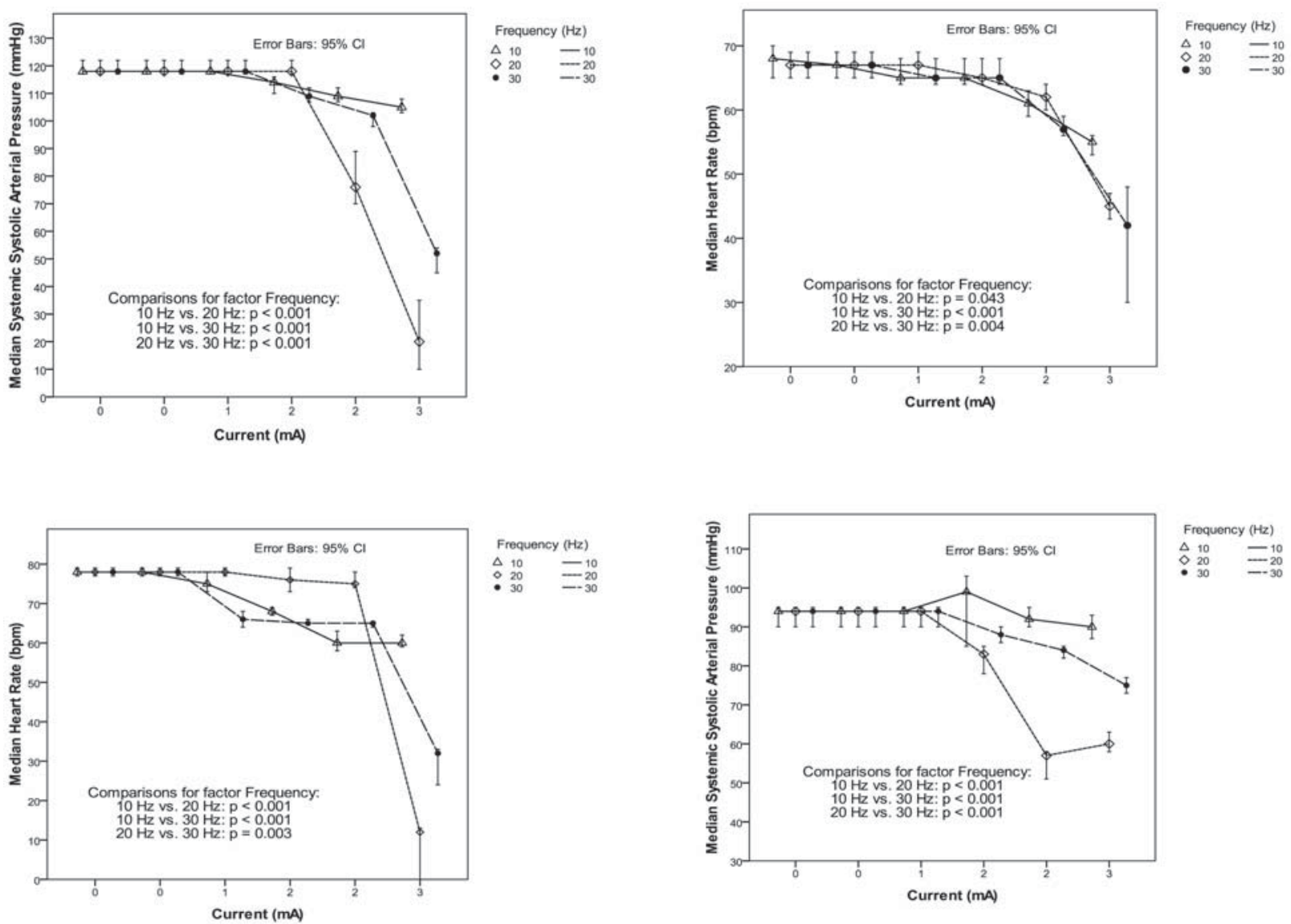

Figure 1. Effect of selective vagus nerve stimulation on heart rate and systemic systolic arterial pressure in the first patient.

Figure 2. Effect of selective vagus nerve stimulation on heart rate and systemic systolic arterial pressure in the second patient.

is composed of different fiber types. (7) The ability to selectively stimulate certain superficial compartments of peripheral nerves has been demonstrated by several authors. (8-11) We have previously reported that selective VNS in humans is possible. (12) However, detailed human data on selective biphasic VNS and its effects on heart rate and systemic arterial pressure are not available. The aim of the present study was to find a clinically acceptable selective biphasic VNS at different stimulation frequencies, which could allow gradual regulation of heart rate and systemic arterial pressure.

\section{Methods}

Two patients undergoing carotid endarterectomy and coronary by-pass surgery were included in the study. The study was approved by The National Medical Ethics Committee, Ministry of Health, Republic of Slovenia (No. 142/02/07, 13 February 2007). Before any trial activity was performed, Informed Consent in accordance with the Informed Consent Procedure was properly obtained.

\section{Anesthesia}

One hour before surgery, patients were premedicated orally with diazepam 5 mg (Apaurin, Krka, Slovenia) and pantoprazole $40 \mathrm{mg}$ (Controloc, Altana Pharma AG, Germany). For induction into anesthesia, the short-acting intravenous anesthetic agent propofol (Propofol 1\% Fresenius, Fresenius Kabi, Austria) was used in doses $1-2$ $\mathrm{mg} / \mathrm{kg}$ of body weight (BW) administered as slow bolus doses (20 - $30 \mathrm{~s}$ ), while remifentanyl (Ultiva, GlaxoSmithKline, UK), as a short-acting synthetic $\mu$-opioid agonist, was used in an initial loading dose of $1-1.5 \mu \mathrm{g} / \mathrm{kg}$ of BW administered in a 30 to $60 \mathrm{~s}$ period. To facilitate endotracheal intubation a dose of 80 to $100 \mu \mathrm{g} / \mathrm{kg}$ of BW of the muscle relaxant vecuronium bromide (Norcuron, Organon, Oss, Holland) was administered intravenously. For maintenance of general anesthesia an inhalation anesthetic isoflurane (Forane, Abbott Laboratories, Queenborough, UK) was used in inspired concentrations of 1.5 to $3.0 \%$, and analgesia was assured by a continuous infusion of $0.3-0.5 \mu \mathrm{g} / \mathrm{kg} / \mathrm{min}$ of remifentanyl. Vecuronium bromide was administered if muscle relaxation of a higher degree was needed for any reason. Low-flow anesthetic technique was performed by administering continuously inhaled fresh gas, flow up to $1.5 \mathrm{~L} / \mathrm{min}$ of oxy- 
gen and air mixture, until reaching 40\% of inspiratory fraction of oxygen $\left(\mathrm{FiO}_{2}\right)$. Patients were mechanically ventilated with a predetermined tidal volume (5 $-7 \mathrm{ml} / \mathrm{kg}$ of body weight), positive endexpiratory pressure between $3-5 \mathrm{~cm}$ $\mathrm{H}_{2} \mathrm{O}$ and respiratory rate between 12 and 15 per minute to maintain end-tidal $\mathrm{CO}_{2}$ concentration between 30 and 35 $\mathrm{mm} \mathrm{Hg}$.

Depth of anesthesia was corrected according to bispectral index values which were maintained between 35 and 40. Two bispectral index monitors and two bispectral index sensors (A-2000, Aspect Medical Sensors, Newton, MA, USA) were placed on the patient's forehead. (13)

Electrocardiogram (ECG) recording ECG from the bipolar limb lead was recorded via self-adhesive, disposable and pre-gelled Ag/AgCl Monitoring Electrode (Premier Skintact ECG electrodes, Leonhard Lang $\mathrm{GmbH}$, Innsbruck, Austria). The ECG signal was delivered to a custom designed bio-amplifier with the following settings: gain 1000 (adjusted to give clean signal with no clipping or saturation visible), $0.1 \mathrm{~Hz}$ low filter, and $150 \mathrm{~Hz}$ high filter.

\section{Hemodynamic monitoring}

Pressure at airway opening was monitored using a transducer for invasive blood pressure monitoring (DPT-6000, PvB-Critical care, Smiths Medical Deutschland $\mathrm{GmbH}$, Germany) and attached to a cuffed endotracheal tube. Invasive systemic arterial pressure was measured by the same transducers attached to an intra-arterial line placed in the left radial artery. The Swan-Ganz catheter (Swan-Ganz CCOmbo Pulmonary Artery Catheter and Vigilance II Monitor, Edwards Inc., USA) for continuous cardiac output, pulmonary artery pressure and central venous pressure measurements was inserted after induction into general anesthesia via the right jugular internal vein.

Design and implantation of a multielectrode spiral cuff

The self-sizing spiral design was chosen for the cuff. A detailed description of the 39-electrode cuff, having thirteen circumferential groups of three electrodes (GTE 1-13) and fabrication can be found in our previous report. (14) The cuff is $18 \mathrm{~mm}$ in length and $2.5 \mathrm{~mm}$ in diameter (inner diameter of the first layer) and has 2.25-2.75 turns, snugly fitting the nerve in its resting position.

\section{Cuff implantation}

After left internal carotid endarterectomy and off-pump coronary artery by-pass surgery the cuff was implanted around the left vagus nerve. The vagus nerve was identified in the carotid sheath in a posterior groove between the carotid artery and the jugular vein. Vessel loops were used to mobilize the vagus nerve so that about 2.5 to $3 \mathrm{~cm}$ were available for attachment of the spiral cuff. Afterwards, the nerve segment was completely freed from its surrounding tissues and the cuff was then carefully attached to the exposed nerve. Since the need to "seal" the cuff on the nerve with a suture was unnecessary, cuff implantation was a relatively simple operation. Namely, the self-fitting nature of the spiral design makes it easy to snuggly place the cuff on the nerve. The spiral cuff design was devised so as to induce very low pressures when installed on a nerve, thus eliminating any passively-induced nerve damage. Special care was taken to route the leads to the connector in order to avoid mechanical tension being transmitted to the cuff. Further precautions were taken during surgery to avoid causing mechanical trauma to the recurrent laryngeal nerve which comes off the left vagus nerve, avoiding potential hoarseness as a permanent sequel after the surgical procedure.

\section{Vagus nerve stimulation}

Stimulus. The electrical stimulus was a combination of quasi-trapezoidal cathodic and rectangular anodic current pulses. Precisely, the resulting current, a biphasic and charge balanced combination was composed of a quasi-trapezoidal cathodic phase with a square leading edge with different intensity, a plateau of $300 \mu \mathrm{s}$, and exponentially decaying phase of $500 \mu \mathrm{s}$, followed by a wide rectangular anodic phase of a low current magnitude. Anodic phase was one-tenth of the magnitude in the cathodic phase. The width of the anodic phase was dependent upon the charge injected in the cathodic phase: the greater the charge, the wider the anodic phase.

Identification of Particular Nerve Compartments and groups of tree electrodes.

Stimuli with intensity of a quasi-trapezoidal cathodic phase of $2 \mathrm{~mA}$ with frequency of $20 \mathrm{~Hz}$ were delivered for 10 seconds quasi-bipolarly to all 13 GTEs within the cuff.

Stimulation of separate GTE regions. The GTE that elicited the largest change in heart rate, namely GTE1, was considered as relevant for further stimulation.

\section{Vagus stimulation with differing} frequency and increasing current.

10-second long pulse trains with intensities of $0.5,1.0,1.5,2.0$ and $2.5 \mathrm{~mA}$ at different frequencies stimulation $(10 \mathrm{~Hz}, 20 \mathrm{~Hz}$ and $30 \mathrm{~Hz})$ were delivered to the before mentioned GTE1 until the absence of cardiac contraction occurred. VNS was terminated instantly after the absence of cardiac contraction was noticed. The time interval between two different stimulations lasted until heart rate or systemic systolic arterial pressure had not reached basal values before stimulation train. At least five equal pulses were applied to analyze the effect on heart rate and arterial pressure.

\section{Data acquisition}

All signals were amplified by a custom designed battery powered bridge amplifier with an adjustable gain. All amplified signals were then fed to a Multifunctional I/O high-performance data acquisition device (NI USB 6259, 16 Inputs, 16 bit, 1.25Ms/s, National Instruments, Austin, Tx, USA). All signals were sampled at $5 \mathrm{kHz}$ using LabView 8.0 for WindowsXP (National Instruments, Austin, Tx, USA). Data were stored on a personal computer hard drive for off-line later analysis.

\section{Statistics}

Two way Analysis of Variance, with Holm-Sidak method for multiple comparisons, was used to analyze effects of different stimulation frequencies and 
currents on heart rate and systolic arterial pressure in individual patients (SigmaPlot 11, Sigma Plot, Chichago, IL, USA). $P$ value $<0.05$ was statistically significant.

\section{Results}

\section{The first patient}

A 62 year old female, one month after anterior wall non-ST elevation myocardial infarction and a 15 year history of arterial hypertension, was the first patient included in our study. Two years before inclusion she had a percutaneous coronary intervention and stent implantation in the left coronary artery due to angina pectoris. Diagnostic evaluation showed important 3 vessel coronary artery disease and significant stenosis of the left internal carotid artery. Echocardiography showed mild left ventricular hypertrophy, normal left ventricular volume, mild systolic dysfunction and mild diastolic dysfunction. Valvular function was normal. She had mild, clinically unimportant pulmonary hypertension at rest. Her basal therapy was: carvedilol $6.25 \mathrm{mg}$ bid, perindopril $2 \mathrm{mg}$ qd, trimetazidine MR $35 \mathrm{mg}$ bid, rosuvastatin $10 \mathrm{mg}$ qd, esomeprazole $40 \mathrm{mg}$ qd, alprazolam $0.25 \mathrm{mg}$ qd, aspirin $100 \mathrm{mg}$ qd. She was referred for endarterectomy of the left internal carotid artery and off-pump coronary artery by-pass graft surgery. Before starting VNS the patient was hemodynamically stable (heart rate 77 bpm, systemic arterial pressure 140/70 $\mathrm{mmHg}$, pulmonary artery pressure $35 / 18 \mathrm{mmHg}$, central venous pressure $10 \mathrm{mmHg}$ and pressure at airway opening $15 \mathrm{mmHg}$ ). Changes of heart rate and systemic systolic arterial pressure during VNS are presented in figures 1. VNS at $10 \mathrm{~Hz}$ provoked the most gradual decrease in the heart rate compared to 20 and $30 \mathrm{~Hz}$ VNS $(p<0.001)$. VNS at $10 \mathrm{~Hz}$ provoked the most gradual decrease of systolic arterial pressure compared to 20 and $30 \mathrm{~Hz}$ VNS ( $p<$ $0.001)$. Ventricular asystole was provoked by $2 \mathrm{~mA} 20 \mathrm{~Hz}$ VNS; atrio-ventricular block (2:1) was detected at $2 \mathrm{~mA}$ with $30 \mathrm{~Hz}$ VNS and at $2.5 \mathrm{~mA}$ with $10 \mathrm{~Hz}$ VNS. VNS was terminated immediately when ventricular asystole or atrio-ventricular block were detected. The heart rate increased instantaneously after termination of VNS. After the procedure, there was no clinically detectable recurrent laryngeal nerve injury.

\section{The second patient}

A 61 year old male was admitted due to unstable angina pectoris. His medical history was significant for arterial hypertension of 18 years. Diagnostic evaluation showed important 3 vessel coronary artery disease and significant stenosis of the left internal carotid artery. Echocardiography showed mild left ventricular hypertrophy, normal left ventricular volume, normal systolic function and mild diastolic dysfunction. Valvular function was normal. He had no pulmonary hypertension. His basal therapy was: carvedilol $3.125 \mathrm{mg}$ bid, atorvastatin $10 \mathrm{mg}$ qd, aspirin 100mg qd. He was referred for endarterectomy of the left internal carotid artery and off-pump coronary artery by-pass graft surgery. Before beginning VNS the patient was hemodynamically stable (heart rate $67 \mathrm{bpm}$, systemic arterial pressure 135/68 mmHg, pulmonary artery pressure 25/16 $\mathrm{mmHg}$, central venous pressure $8 \mathrm{mmHg}$ and pressure at airway opening $15 \mathrm{mmHg}$ ). Changes of heart rate and systemic systolic arterial pressure during VNS are presented in figures 2. VNS at $10 \mathrm{~Hz}$ provoked the most gradual decrease in heart rate compared to 20 and $30 \mathrm{~Hz}$ VNS $(\mathrm{p}<0.05)$. VNS at $10 \mathrm{~Hz}$ provoked the most gradual decrease of systolic arterial pressure compared to 20 and $30 \mathrm{~Hz}$ VNS $(p<0.001)$. VNS provoked no devastating heart conduction abnormalities. After the procedure, there was no clinically detectable recurrent laryngeal nerve injury.

\section{Discussion}

In our human case study, the frequency and current dependant effects of selective VNS on heart rate and systemic systolic arterial pressure are reported. The most profound effects were detected when using $20 \mathrm{~Hz}$ stimulation. On the other hand, $10 \mathrm{~Hz}$ stimulation offers more gradual heart rate and systolic arterial pressure control and could clinically be more appropriate. Our work is based on previous studies which demonstrated that the human heart receives vagal postganglionic fibres from specific superficial compartments of the left vagus nerve and that these fibres are organized longitudinally along the length of the nerve. (15) It was shown that most of the fibers that comprise the human vagus nerve are small-diameter non-myelinated C-fibres; the rest are intermediate-diameter myelinated B-fibres and large-diameter myelinated A-fibres. (7) Most of the A-fibres, and many of the B-fibres, are given off in the cervical branches of the vagus nerve. A and B-fibres, are primarily cardiovascular and respiratory neurons. (16-19) Different types of nerve fibres respond differently to various types of stimulating pulses. (8-11) To stimulate mostly B-fibres, the combination of quasitrapezoidal cathodic and rectangular anodic current pulses, which we used in our study, was previously proposed. (20) It is assumed that propagation of action potential of A-fibres could be blocked by hyperpolarization at short-circuited anodes while activation of C-fibres could be abolished. To increase the parasympathetic stimulation of the heart, the number of fibres not blocked should be progressively increased by decreasing the magnitude of the current, applied by short-circuited anodes. As a result, the parasympathetic stimulation of the heart is progressively increased in a smaller-to-larger diameter fiber order, mimicking the body's natural method of increasing stimulation. Such stimulation enables the use of a cathodic current sufficiently weak to avoid stimulation of $\mathrm{C}$ fibres near the surface of the nerve, while still sufficiently strong to stimulate B fibres, including B fibres situated more deeply in specific compartments of the nerve. Stimulation of C-fibres is to be avoided because these fibres transmit pain sensations and are important for regulation of reflexes such as respiratory reflexes. Furthermore, by not stimulating 
A-fibres, adverse effects sometimes associated with dyspnea and hoarseness could be avoided. (21) Other side effects of non-selective vagus nerve stimulation are also described. (22) After our experimental protocol, no clinically important vagus nerve dysfunction was noticed. We used a special cuff design, as published previously. (14) Although cuffs are not entirely free from problems, their advantage is that the reproducibility of selective stimulations is superior. (23) Namely, the cuff can conform to the shape of the nerve, providing a low stimulation threshold by allowing a large stimulation contact area with the nerve. Furthermore, confinement of electric current within the cuff is maximized with the spiral design. Our human case study has at least 3 major limitations. First, only two patients were included and they were already on a beta blocking drug (carvedilol). Carvedilol could potentially influence our results. The first patient, in whom AV block and asystole were provoked, was on a higher dose of carvedilol than the second patient. Second, trains of stimuli were not ECG gated, so it is impossible to analyze the time from the beginning of the stimulus to the time of the effect. Third, in our report only acute effects were studied. It is not known how stimulation threshhold and stimulation efficiency could change with prolonged implantation. We have previously shown that in dogs prolonged implantation and stimulation through the same cuff design, as in the current study, were associated with a buildup of connective tissue encapsulation around the cuff as well as within the cuff. (24) It was also observed that there was some mechanical distortion of the nerve and neural damage. However, functional deficits were not observed. It can be concluded that selective VNS with trains of stimuli (combination of quasi-trapezoidal cathodic and rectangular anodic current pulses) produces frequency and current dependant effects on heart rate and systolic arterial pressure. The most profound effects are detected when using the $20 \mathrm{~Hz}$ stimulation. However, the $10 \mathrm{~Hz}$ stimulation offers more gradual heart rate and systemic systolic arterial pressure control and could be clinically more interesting. Selective VNS could in future be used for controlling heart rate during atrial fibrillation, heart failure, surgery and in critically ill patients.

\section{ACKNOWLEDGMENTS}

We thank the doctors, nurses and technical support staff for their assistance in this study. This study was in part supported by the Ministry of Education and Science, Republic of Slovenia (Research Programs P3-0171, P3-0331, P3-0043).

\section{REFERENCES}

1. Carlsten A, Folkow B, Hamberger CA.Cardiovascular effects of direct vagal stimulation in man. Acta Physiol Scand 1957;41:68-76.

2. Henning RJ, Levy MN. Effects of autonomic nerve stimulation, asynchrony, and load on $\mathrm{dP} / \mathrm{dtmax}$ and on $\mathrm{dP} / \mathrm{dtmin}$. Am $\mathrm{J}$ Physiol 1991;260: H1290-8.

3. Xenopoluos NP, Applegate RJ. The effect of vagal stimulation on left ventricular systolic and diastolic performance. Am J Physiol 1994;266:H2167-73

4. Levy MN, Martin PJ. Neural Regulation of the Heart Beat. Annu Rev Physiol 1981;43:443-53.

5. Lewis ME, Al-Khalidi AH, Bonser RS. Vagus nerve stimulation decreases left ventricular contractility in vivo in the human and pig heart. $\mathrm{J}$ Physiol 2001; 534:547-52.

6. Matheny RG, Shaar CJ. Vagus nerve stimulation as a method to temporarily slow or arrest the heart. Ann Thorac Surg 1997;63:S28-9.

7. Chase MR. An Experimental Study of the vagus nerve. J Compar Neurol 1996;26:421-8.

8. Sweeney JD, Ksienski DA, Mortimer JT. A nerve cuff technique for selective excitation of peripheral nerve trunk regions. IEEE Trans Biomed Eng 1990;37:706-15.

9. Veraart C, Grill WM, Mortimer JT. Selective control of muscle activation with a multipolar nerve cuff electrode. IEEE Trans Biomed Eng 1993;46:640-53.

10. Goodall EV, de Breij JF, Holsheimer J. Position-selective activation of peripheral nerve fibres with a cuff electrode. IEEE Trans Biomed Eng 1996;43:851-6.

11. Choi AQ, Cavanaugh JK, Durand DM.Selectivity of Multiple-Contact Nerve Cuff Electrodes: A Simulation Analysis. IEEE Trans Biomed Eng 2001;48:165-72

12. Rozman J, Pečlin P, Kneževič I, Mirkovič T, Podbregar M. Heart function influenced by selective mid-cervical left vagus nerve stimulation in a human case study. Hypertens Res 2009;32:1041-3. 
13. Hemmerling TM, Olivier JF, Basile F, Le N, Prieto I. Bispectral index as an indicator of cerebral hypoperfusion during off-pump coronary artery bypass grafting. Anesth Analg 2005;100:354-6.

14. Rozman J, Bunc M. Modulation of visceral function by selective stimulation of the left vagus nerve in dogs. Exp Physiol 2004;89:717-25.

15. Sunderland S, Bedbrook GM. The cross-sectional area of peripheral nerve trunks occupied by the fibres representing individual muscular and cutaneous branches. Brain 1949;72:613-24.

16. Rossi L, Nappo A. Subdivisions of the extrinsic cardiac nervous system (mediastinal plexi, intercarotid receptors and bulbar center). Pathologica 1994;86:441-2.

17. Zagon A, Ishizuka K, Rocha I, Spyer KM. Slow vagal inhibition in neurones of the ventrolateral medulla oblongata of the rat: a possible mechanism for tonic, vagally evoked sympatho-inhibition. J Hum Hypertens 1997;11:599-600

18. Spyer KM, Lambert JH, Thomas T. Central nervous system control of cardiovascular function: neural mechanisms and novel modulators. Clin Exp Pharmacol Physio 1997;24:743-7.

19. Hoffman HH, Schnitzlein HN. The numbers of nerve fibres in the vagus nerve of man. Anat Rec 1961;139:429-35.

20. Fang ZP, Mortimer JT. Selective activation of small motor axons by quasitrapezoidal current pulses. IEEE Trans Biomed Eng 1991;38:168-79.

21. Zumstag D, Jenny D, Wieser HG. Vocal cord adduction during vagus nerve stimulation treatment of epilepsy. Neurology 2000;54:1388-9.

22. Hatton KW, McLarney JT, Pittman T, Fahy BG. Vagus nerve stimulation: overview and implications for anesthesiologists. Anast Analg 2006;103:1241-9.

23. Tarver WB, George RE, Maschino SE, Holder LK, Wernicke JF. Clinical experience with a helical bipolar stimulating lead. Pacing Clin Electrophysiol 1992:15:1545-56

24. Rozman J. Tissue response to chronic selective stimulation of vagus nerve of a dog using multi-electrode spiral cuff. Period Biol 2006;108:2-9. 